\title{
Análise Dimensional de um Escoamento em Leito Poroso
}

\author{
Medeiros, B.1; Abreu, C.E. ; Schiaffino, M.S. ; Martins, M. F.1 \\ 1 Programa de Pós-Graduação em Engenharia Mecânica, Universidade Federal do Espírito Santo, Vitória, ES, Brasil
}

\begin{abstract}
Resumo
Atualmente, o estudo termo-fluidodinâmico de escoamentos reais apresenta pouquíssimas soluções puramente por métodos analíticos. O estudo desses escoamentos depende intimamente das relações empíricas obtidas por resultados experimentais. O método de análise mais usual para a solução desse tipo de problema consiste inicialmente, na análise analítica, obtendo um modelo matemático simples o suficiente para fornecer uma solução ao problema. A partir desse modelo, são realizados experimentos visando o refino desse modelo matemático. Assim, descreve-se a interface entre modelos empíricos e analíticos. Entretanto, o estudo de um fenômeno realizando experimentos com parâmetros fixos pode ser extremamente demorado e trabalhoso. Existem vários modelos matemáticos empregados nessa aplicação. Este trabalho visa utilizar a equação de Ergun, um dos modelos mais utilizados nessa aplicação.
\end{abstract}

Palavras-chave: Gaseificação, Biomassa, Adimensionalização.

\section{Introdução}

Supondo um experimento de um fenômeno que dependa de cinco parâmetros, para obter resultados o suficiente a fim de descrever um comportamento do fenômeno em função de um dos parâmetros, suponha que seriam necessários 10 testes. Desta forma, seriam necessários 100.000 testes para descrever o comportamento do parâmetro em questão, inviabilizaria esse estudo devido ao tempo necessário para realizar todos os experimentos.

Visando reduzir esse tempo de experimentos, pode-se realizar uma Análise Dimensional do fenômeno em questão, criando uma relação entre os cinco parâmetros e assim, reduzindo drasticamente o número de experimentos a serem realizados para descrever 0 fenômeno.

Assim, pode-se afirmar que a Análise Dimensional de um fenômeno, é usada para facilitar a sua compreensão em função dos parâmetros as qual essa depende.

\section{Metodologia}

Para realizar a adimensionalização do fenômeno em questão, será utilizado o Teorema dos $\mathrm{Pi}$ de Buckingham.

Inicialmente, temos um parâmetro dependente que pode ser expresso em função de $n-1$ parâmetros independentes como mostrado a seguir.

$$
q_{1}=f\left(q_{2}, q_{3}, q_{4}, \ldots, q_{n}\right.
$$

Onde:

$$
\begin{aligned}
& q_{1} \text { : parâmetro dependente } \\
& q_{2} \text { até } q_{n-1} \text { : parâmetros independentes }
\end{aligned}
$$

Matematicamente, pode-se transformar a equação $1 \mathrm{da}$ seguinte forma:

$$
g\left(q_{1}, q_{2}, q_{3}, \ldots, q_{n}\right)=0
$$

Onde g é uma função arbitrária, diferente de f.
O Teorema dos Pi de Buckingham diz que para uma relação como a equação 2 , os $n$ parâmetros podem ser agrupados em $n-m$ parâmetros adimensionais expressos na forma funcional da seguinte forma:

$$
G\left(\pi_{1}, \pi_{2}, \ldots, \pi_{n-m}\right)=0
$$

ou

$$
\pi_{1}=G_{1}\left(\pi_{2}, \pi_{3}, \ldots, \pi_{n-m}\right)
$$

Onde $\mathrm{m}$ é, usualmente, igual ao número mínimo de dimensões independentes necessárias para especificar as dimensões dos $\mathrm{n}$ parâmetros independentes.

Infelizmente, o teorema não obtém a fórmula analítica das funções $G$ e G1. Essas devem ser determinadas empiricamente e para isso se recomenda utilizar um algoritmo adequado obter a equação funcional do fenômeno a ser estudado em função de parâmetros adimensionais.

Atualmente a equação de Ergun é o modelo mais usado e aceito para a previsão da queda de pressão em leitos fixos. E aplicada largamente e sem restrições de número de Reynolds, tanto para leitos de partículas uniformes quanto não uniformes, e até mesmo para misturas de diversos tamanhos de partículas. (Figura № 1).

Equação de Ergun:

$\frac{-\Delta P}{L}=150 \frac{(1-\varepsilon)^{2} \mu}{\Phi_{p}^{2} \varepsilon^{3} d_{p}^{2}}+1,75 \frac{(1-\varepsilon) \rho_{f}}{\Phi_{p} \varepsilon^{3} d_{p}}$

onde

$-\triangle P$ : queda de pressão através do leito

$L \quad$ : percurso realizado no leito poroso

$\varepsilon$ : porosidade

$\mu \quad$ : viscosidade do fluído

$\Phi_{p}$ : esfericidade

$d_{p}$ : diâmetro da partícula

$\rho_{f} \quad$ : massa específica do fluído 


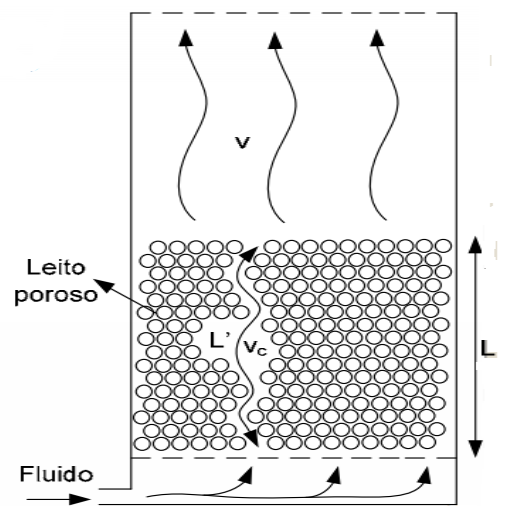

Figura №1: Deslocamento do fluído no reator

A partir do Teorema dos $\mathrm{Pi}$ de Buckingham obteve os grupos adimensionais para um escoamento em meio poroso e adimensionalizou a equação de Ergun.

As equações de 5 a 9 apresentam os grupos adimensionais encontrados no escoamento em leito poroso.

$$
\begin{aligned}
& \Pi_{1}=\frac{\Delta p}{\rho v^{2}} \\
& \Pi_{2}=\frac{\mu}{d_{D^{v \rho}}} \\
& \Pi_{3}=\frac{L}{d_{\nu}} \\
& \Pi_{4}=\epsilon \\
& \Pi_{5}=\phi
\end{aligned}
$$

Então a equação de Ergun adimensionalizada se torna: $\frac{\Delta P}{\rho v^{2}}=150 \frac{L}{d_{p} \operatorname{Re}} \frac{(1-\epsilon)^{2}}{\phi^{2} \epsilon^{3}}+1,75 \frac{L}{d_{p}} \frac{(1-\epsilon)}{\phi \epsilon^{3}}$

\section{Resultados}

$\mathrm{Na}$ análise dos parâmetros adimensionais verifica-se na figura №2 que o aumento do número de Reynolds diminui o adimensional $\frac{\Delta P}{\rho v^{2}}$, isto é, a pressão dinâmica passar a ser o fenômeno mais evidente no processo.

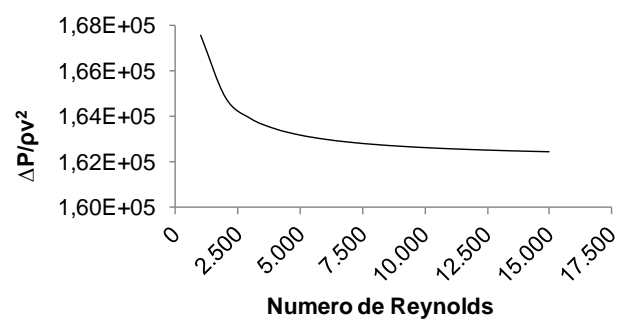

Figura №2: Analise do escoamento em função de Reynolds.

Em relação à porosidade (figura №3), o seu aumento no leito facilita o escoamento, com era de se esperar.

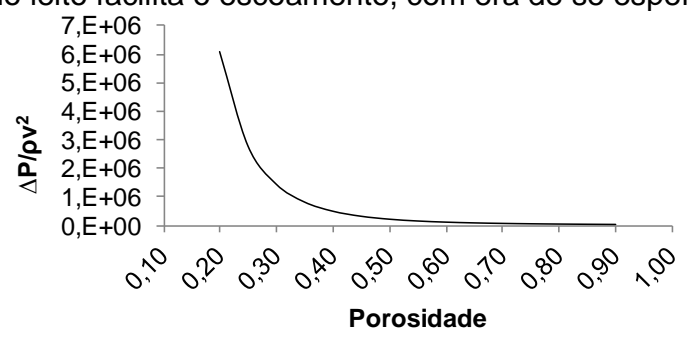

Figura №3: Análise do escoamento em função da porosidade para Reynolds baixa $(R e=100)$.

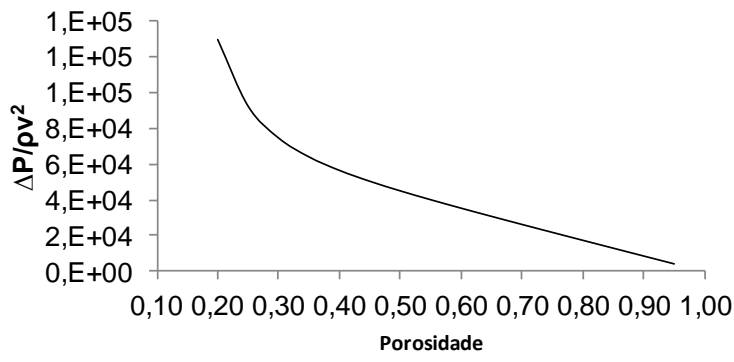

Figura №4: Análise do escoamento em função da porosidade para Reynolds alto $(R e=10000)$.

O aumento do parâmetro adimensional L/dp prejudica o escoamento do leito poroso, também conforme se prevê na literatura.

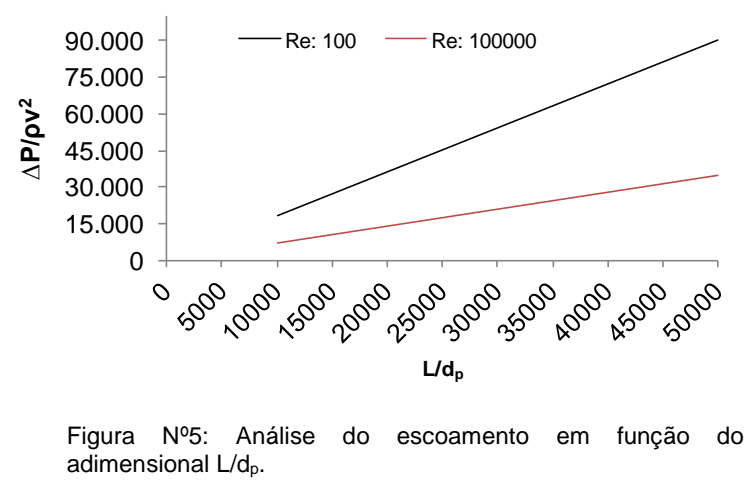

\section{Conclusão}

Conclui-se que a aplicação do Teorema dos $\mathrm{Pi}$ de Buckingham reduz o número de parâmetros no problema analisado e consequentemente facilita a interpretação dos resultados.

\section{Referências}

MOTTA, E.P. Queda de pressão em um leito de partícula de xisto. Dissertação de mestrado. UFPR. Paraná. 2009. 132p.

FOX, W.R.; MCDONALD, T.A. Mecânica dos fluidos. Rio de Janeiro. $5^{\circ}$ ed. $519 p$.

JIA Y.; LI J.; HLAVKA D. Flow through packed beds. 2009. 